\title{
RESUMEN.
}

El propósito de esta investigación, es realizar un análisis descriptivo de los posibles efectos que se persiguen con la aplicación de la Norma de Carácter General (NCG) número 385 de la Superintendencia de Valores y Seguros, y los beneficios que esta traería a las Sociedades Anónimas Abiertas del sector retail, que difunden de manera pública las prácticas de gobierno corporativo. Esto se realizó mediante un análisis documental, desarrollado bajo un paradigma cualitativo con alcance comprehensivo, con la finalidad de analizar las prácticas que permiten el fortalecimiento de los gobiernos corporativos de las sociedades anónimas abiertas del sector retail en Chile, mediante el análisis de aplicación de la NCG No 385 . De la información obtenida, se concluyó que la mayoría de las empresas de retail, reguladas por la SVS, cumplen con emitir el informe sobre prácticas de gobierno corporativo a pesar de no ser algo obligatorio, las prácticas que más cumplen las empresas de retail, son las relacionadas con el control y gestión de riesgo.

Palabras Claves: Gobiernos Corporativos, retail, Superintendencia de Valores y Seguros, Sociedad Anónima.

\section{ANÁLISIS DE LAS PRÁCTICAS PARA EL FORTALECIMIENTO DE GOBIERNO CORPORATIVO, ESTABLECIDAS EN LA NORMA DE CARÁCTER GENERAL No 385 , EMITIDA POR LA SUPERINTENDENCIA DE VALORES Y SEGUROS EL AÑO 2015. APLICADO AL SECTOR RETAIL EN CHILE. \\ Dominique Garrido Araya / Tripcy Briceño Tello²}

\section{ANALYSIS OF THE PRACTISES FOR THE STRENGHTHENING OF CORPORATE GOVERNANCE ESTABLISHED IN THE GENERAL STANDARD $N^{\circ} 385$ ISSUED BY THE SUPERINTENDENCY OF SECURITIES AND INSURANCE IN THE YEAR 2015. APPLIED TO THE RETAIL SECTOR IN CHILE.}

\section{ABSTRACT}

This research aims to make a descriptive analysis of the possible effects, pursued with the application of the General Standard Number 385 of the Superintendency of Securities and Insurance, and benefits which the latter would entail to open corporations belonging to the retail sector which publicly spread corporate governance practices. This will be achieved through a documental analysis, developed under a qualitative paradigm with comprehensive meaning, with the aim of analyzing those practises which allow the strenghthening of corporate governance within open corporations of the retail sector in Chile by means of an analysis of the application of the $\mathrm{N}^{\circ} 385$. From the obtained data, it was concluded that most of the retail companies, supervised by the SVS, fulfill with the requirement of issuing the report about corporate governance practices although it is not something compulsory. In other respects, the practices that retail companies most fulfill are those related to control and risk management.

Key Words: Corporate Governance, retail, Superintendency of Securities and Insurance, Corporation.

\footnotetext{
${ }^{1}$ Contador Público Auditor, Licenciado en Sistemas de Información Financiera y Control de Gestión, Escuela de Auditoría, Universidad de Valparaíso. dominique.garrido@uv.cl

${ }^{2}$ Contador Público Auditor, Licenciado en Sistemas de Información Financiera y Control de Gestión.
} 
Revista de Investigación Aplicada en Ciencias Empresariales 


\section{PROBLEMA}

Actualmente los mercados deben operar en un escenario en donde se ha instalado la desconfianza, ya que en el último tiempo se ha gatillado en mayor número los casos de corrupción, fraudes y escándalos financieros, en que se han visto involucradas empresas chilenas, entre ellas La Polar. Por lo cual, se han tratado de buscar medidas que dirijan a las organizaciones al fiel cumplimiento de las normas y no conduzcan a casos como los mencionados anteriormente, lo que ha conllevado a instalar Gobiernos Corporativos en las empresas, principalmente en las Sociedades Anónimas Abiertas.

En relación a lo anterior la Superintendencia de Valores y Seguros (SVS), el 8 de junio del 2015, emitió las Normas de Carácter General (NCG) No 385 y 386 las cuales reemplazan o modifican a las NCG No 341 y №30, respectivamente, con el fin de perseguir el fortalecimiento del Estándar de Gobiernos Corporativos de las Sociedades Anónimas Abiertas, para mejorar la confianza en los mercados por parte de inversionistas, proveedores, competidores, empleados y demás grupos de interés en la sociedad.

\section{REVISIÓN DE LITERATURA}

\section{Antecedentes generales}

Actualmente en Chile se ha generado un fenómeno empresarial que permite un mejor control al interior de las organizaciones, esto es la incorporación de los gobiernos corporativos, si bien esta no es una figura nueva como herramienta de gestión, ya que siempre ha existido, ha retomado el protagonismo dado el actual escenario político, económico y financiero que se han evidenciado en Chile en el último tiempo, puesto que determinadas corporaciones han realizado malas prácticas empresariales como son la corrupción, cohecho, colusión, fraudes fiscales, escándalos financieros, entre otros, que finalmente detono una crisis de desconfianza tanto en el modus operandi de las empresas y que repercuten en un descontento social.

\section{Gobierno Corporativo}

A nivel internacional se encuentra la definición entregada por la Organización para la Cooperación y Desarrollo Económicos (OCDE), la cual "sostiene que el gobierno corporativo es el mecanismo de los accionistas que sirve de contrapeso y guía a la administración del negocio, para asegurar la eficiencia, la equidad, la transparencia y los buenos resultados, con razonabilidad, rendición de cuentas, transparencia y responsabilidad". (Organización para la Cooperación y Desarrollo Económico, 2015: 1) 


\section{Funciones de los Gobiernos Corporativos}

La función principal del gobierno corporativo es permitir la viabilidad de la empresa a largo plazo, para estos debe fundarse en principios claros y estables.

\section{Objetivos del Gobierno Corporativo}

Los principales objetivos de los gobiernos corporativos son:

- Velar por la transparencia,

- permitir el conocimiento de cómo los directivos gestionan los recursos,

- $\quad$ proveer de instrumentos de resolución de conflictos de interés entre los distintos grupos que conforman el gobierno y buscar el logro de equilibrios al interior del sistema. (Wigodski y Zuñiga , 2001)

Estos objetivos ayudan al cumplimiento del objetivo final, el que consiste en contribuir a la maximización del valor de las compañías, en un horizonte de largo plazo.

\section{Evolución de la normativa de los Gobiernos Corporativos}

La normativa que regula los gobiernos corporativos ha ido evolucionando en el transcurso del tiempo, tal como se muestra en la figura $\mathrm{N}^{\circ} 1$. 
Figura №1: Evolución de la normativa de los gobiernos corporativos en Chile

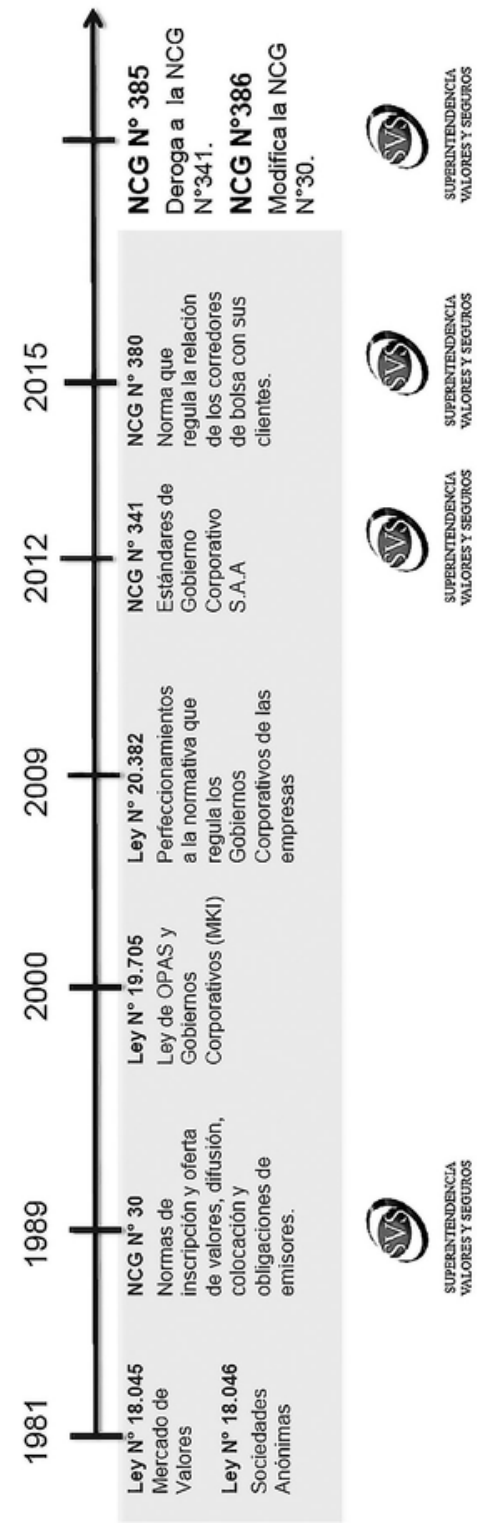

Fuente: Bolsa de Santiago (s.f).

\section{Ley $\mathrm{N}^{\circ} \mathbf{1 8 . 0 4 5}$}

Ley de Mercado de Valores, ofrece el marco normativo por el cual se rigen las Sociedades Anónimas Abiertas en términos de:

- Obligaciones de información continua y reservada que deben cumplir.

- Sus transacciones de valores de oferta pública. 
- Registro y transacción de valores en el mercado secundario.

- Los grupos empresariales, controladores y personas relacionadas a las sociedades.

- Información privilegiada.

\section{Ley $\mathrm{N}^{\circ} \mathbf{1 8 . 0 4 6}$}

Ley de Sociedades Anónimas, entrega el soporte para la constitución, administración y funcionamiento de las Sociedades Anónimas en relación a:

- La sociedad y su constitución.

- Su capital social, de las acciones y los accionistas.

- La administración de la compañía, el directorio y las juntas de accionistas.

- Las responsabilidades, sanciones y operaciones con personas relacionadas a la sociedad.

- Entre otros aspectos.

\section{Norma de Carácter General № 30.}

Norma de inscripción y oferta de valores, difusión, colocación y obligaciones de emisores. Establece los antecedentes e información que las sociedades anónimas deben presentar en términos de:

- $\quad$ Inscribir sus valores públicamente.

- Información obligatoria a ser difundida a la SVS y público en general.

- Requisitos de información para la inscripción de emisión de acciones

- Requisitos de información para la inscripción de emisión instrumentos de deuda.

\section{Ley $N^{\circ} 19.705$}

Ley de Opas y Ggobiernos Corporativos, fomenta la regulación de la oferta pública de adquisición de acciones (OPAS) para la protección de inversionistas minoritarios, además de establecer pautas generales para el régimen de gobierno corporativo de las sociedades anónimas. En ella se establecen:

- Creación Comité de Directores

- Regulación de transacciones entre partes relacionadas

- Extensión de causales que dan derecho a retiro a los inversionistas frente a materias que lo perjudiquen 
- Establecimiento de la acción civil derivada.

\section{Ley $N^{\circ} 20.382$}

Perfeccionamiento a la normativa que regula los gobiernos corporativos de la empresa, para fomentar la protección de los intereses de los accionistas versus la administración de la empresa, disminuir conflictos de interés, fortalecer el rol de las auditorías externas y evitar malas prácticas con el manejo de información privilegiada.

- Fortalecer los directorios de las empresas a través de la designación de al menos un director "independiente".

- Fortalecer la figura de un Comité de Directores, compuesto por mayoría de miembros independiente.

\section{Norma de Carácter General № 341}

Estándares de gobierno corporativo sociedades anónimas abiertas, que fomenta el proveer información adecuada al público inversionista respecto de las políticas y prácticas de gobierno corporativo adoptadas por las sociedades anónimas abiertas. La autoevaluación de las sociedades se realiza en relación a los siguientes aspectos:

- Funcionamiento del directorio.

- Relación entre la sociedad, los accionistas y el público en general.

- Sustitución y compensación de ejecutivos principales.

- Definición, implementación y supervisión de políticas y procedimientos de control interno y gestión de riesgos en la empresa.

- Otras prácticas adoptadas por la sociedad, no referidas en las materias anteriores.

\section{Norma de Carácter General № 380.}

Norma que regula el actuar de los corredores de bolsa, agentes de valores y corredores de productos respecto a su relación con el cliente, estableciendo mayores exigencias en:

- La relación contractual entre intermediario y usuarios.

- Transparencia en la ejecución de las operaciones bursátiles.

- Detección y resolución de conflictos de interés.

- Conocimiento de clientes. 
- Establecimiento de un registro con documentación de respaldo.

\section{Norma de Carácter General № 385}

Normativa que deroga la NCG N³41, establece los siguientes estándares de Gobierno Corporativo:

- Fomentar la adopción de políticas en materia de responsabilidad social y desarrollo sostenible.

- Fomentar la difusión de información a los accionistas y público en general.

- Mejorar la calidad y confiabilidad de la información contenida en la autoevaluación de los directorios.

- Promover la adopción de principios, directrices y recomendaciones nacionales e internacionales.

- Explicitar el tratamiento de los conflictos de interés y los procedimientos de actualización del Código de Conducta del directorio.

\section{Norma de Carácter General N 386}

Normativa que modifica la NCG №30, contempla la incorporación, en la memoria anual de información relativa a materias de responsabilidad social y desarrollo sostenible en los siguientes aspectos:

- Diversidad en el directorio (género, nacionalidad, edad y antigüedad).

- Diversidad en la gerencia general y demás gerencias que reportan a esta gerencia 0 al directorio.

- Diversidad en la organización (género, nacionalidad, edad, antigüedad)

- Brecha salarial por género.

\section{Superintendencia de Valores y Seguros (SVS)}

La Superintendencia de Valores y Seguros (SVS) es una institución autónoma, con personalidad jurídica y patrimonio propio, que se relaciona con el Gobierno a través del Ministerio de Hacienda. Tiene por objeto la superior fiscalización de las actividades y entidades que participan de los Mercados de Valores y de Seguros en Chile. Así, a la SVS le corresponde velar porque las personas o instituciones supervisadas, desde su iniciación hasta el término de su liquidación, cumplan con las leyes, reglamentos, estatutos y otras disposiciones que rijan el funcionamiento de estos mercados. 


\section{Sociedad Anónima}

Tanto la Ley № 18.046, como el Código Civil Chileno entregan la siguiente definición de "La Sociedad Anónima es una persona jurídica formada por la reunión de un fondo común, suministrado por accionistas responsables solo por sus respectivos aportes y administrada por un directorio integrado por miembros esencialmente revocables.

La Sociedad Anónima es siempre mercantil, aun cuando se forme para la realización de negocios de carácter civil." (Ley No 18.046, 2016)

\section{Clases de sociedades anónima}

La misma ley señala que las sociedades anónimas pueden ser de tres clases:

- Sociedad Anónima Abierta: Son aquellas que inscriban voluntariamente 0 por obligación legal sus acciones en el registro de valores. Son sometidas a la fiscalización de la Superintendencia de Valores y Seguros, salvo que la ley lo someta a control de otra superintendencia, en este caso también quedara sometida a la primera, en lo que corresponde, cuando emitiera valores. (Ley № 18.046, 2016)

- Sociedad Anónima Especial: Son las compañías aseguradoras 0 reaseguradoras, las sociedades anónimas administradoras de fondos mutuos, las bolsas de valores y otras sociedades que la ley expresamente somete a normas espaciales, se forman, existen y aprueban por escritura pública, obtención de una resolución de la Superintendencia que autorice su existencia e inscripción y publicación del certificado especial que otorgue dicha Superintendencia. (Ley No 18.046, 2016)

Estas sociedades se regirán por las mismas disposiciones legales y reglamentarias aplicables a las sociedades anónimas abiertas, en todo lo que no se oponga a lo dispuesto en las disposiciones especiales que las rigen. Salvo que las sociedades anónimas especiales sean emisores de valores, no deberán inscribirse en el Registro de Valores de la Superintendencia.

- Sociedad Anónima Cerrada: Son aquellas que no clasifican como sociedades anónimas abiertas 0 especiales. No pueden hacer oferta pública de sus acciones, salvo que se sometan voluntariamente a la fiscalización de la SVS. (Ley No 18.046, 2016)

\section{Retail}

El Retail representa a un sector de la economía, que engloba a todos los tipos de comercio involucrados en la comercialización masiva de productos y/o servicios uniformes, a una cantidad elevada de clientes. El término inglés hace referencia, 
a lo que en español se puede denominar como Comercio Minorista o Comercio Detallista.

Las cadenas que se dedican al sector retail disponen de una amplia estructura. Por un lado, suelen contar con almacenes, depósitos o centros de distribución donde reciben los productos que compran al por mayor. Luego estos productos son enviados a los distintos locales comerciales o sucursales, que constituyen los puntos de venta donde acude el comprador final a adquirir la mercadería. (Leyton, 2013)

\section{Principales formatos de retail}

- Tiendas por departamento: Las tiendas por departamento son establecimientos de grandes dimensiones en los cuales se venden $u$ ofertan una gran variedad de productos que corresponden a diversos usos y prácticas, como confecciones, menaje, decoración, deportes, etc.

- Supermercados: Un supermercado es un establecimiento, de tamaño variable, que vende bienes de consumo. Entre éstos se encuentran: alimentos, ropa, artículos deportivos, artículos de higiene u otros. Se diferencian de las tiendas por departamento porque su centro está en el sector alimenticio.

- Mejoramiento del hogar: Al igual que las tiendas por departamento, las tiendas de mejoramiento del hogar son establecimientos de grandes dimensiones en los cuales se venden u ofertan una gran variedad de productos. Pero en este caso y como su nombre lo indica, estas necesidades se centran en el hogar, la construcción, decoración, mantención y mejoramiento.

\section{PROPUESTA METODOLÓGICA}

La presente investigación se desarrolla bajo un paradigma cualitativo, con un alcance comprehensivo, se basa en un análisis documental, donde se utilizará el método analítico, a través del cual se busca identificar prácticas que permitan el fortalecimiento de Gobiernos Corporativos, a través de la aplicación de la norma de Carácter General n 385 que es llevada a cabo por las sociedades anónimas abiertas chilenas.

\section{ANÁLISIS DE RESULTADOS}

Se seleccionaron las 10 sociedades anónimas del sector retail, que al 31 de marzo del 2016 emitieron el informe sobre prácticas de Gobierno Corporativo correspondiente al año 2015. 
El informe sobre prácticas de Gobierno Corporativo, cuyo formato se encuentra en la norma de Carácter General $n^{0} 385$ consta de 99 ítems, agrupados en 4 grupos, los cuales serán considerados en aquellos puntos más significativos, en los que las empresas concordaban en las respuestas, para señalar si cumplían o no con las prácticas.

El gráfico 1 muestra que todas las empresas bajo estudio, con excepción de Falabella, responden a las mayorías de las prácticas de gobierno corporativo señalando que no las cumplen, siendo La Polar aquella que entrega una mayor cantidad de respuestas negativa $(88,89 \%)$, seguida por Forus $(79,80 \%)$ e Hipermarc con un $78,79 \%$.

\section{Gráfico № 1 Cumplimiento de prácticas de Gobierno Corporativo por empresas} baịo estudio.

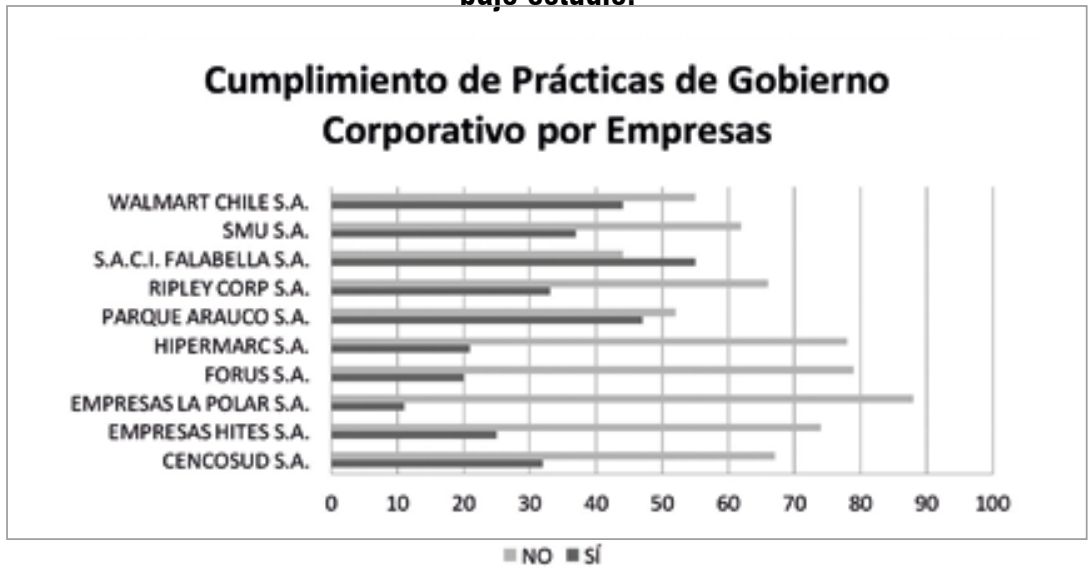

Fuente: Elaboración Propia. Año 2015.

\section{Del funcionamiento y dirección del Directorio.}

Este grupo está compuesto por 51 ítems, donde el $70 \%$ de las respuestas entregadas por las 10 empresas de retail en estudio fue negativa, lo cual se evidencia en el gráfico $\mathrm{N}^{\circ} 2$. 
Gráfico N 2: Cumplimiento de prácticas de Funcionamiento y Dirección del Directorio.

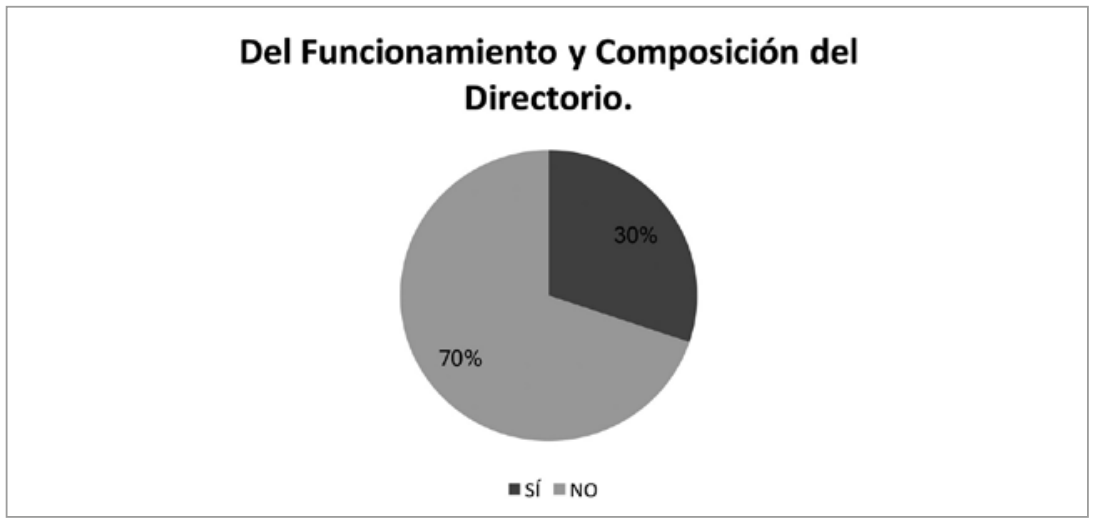

Fuente: Elaboración Propia. Año 2015.

A continuación, el gráfico № 3 muestra el análisis de aquellas prácticas, en el cual el $90 \%$ y más de las empresas concordaron en su respuesta.

Gráfico $\mathrm{N}^{\circ}$ 3: Prácticas relacionadas con el funcionamiento y composición del Directorio.

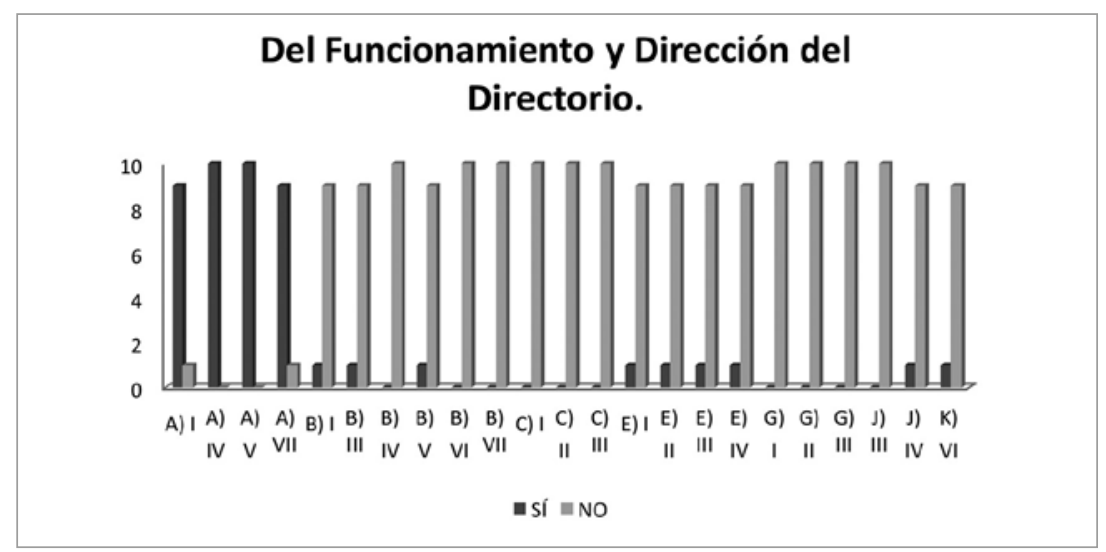

Fuente: Elaboración Propia. Año 2015.

a) El Directorio cuenta con un procedimiento o mecanismo para la inducción de cada nuevo integrante, que tiene por objeto facilitar a éste el proceso de conocimiento y comprensión de:

- Los negocios, materias y riesgo, incluidos los de sostenibilidad, que son considerados más relevante, así como de las razones por las que en opinión del directorio aquéllos tienen esta condición.

La mayoría de las empresas en estudio, cuentan con políticas donde se establece la inducción de nuevos integrantes al directorio. 
- $\quad$ El marco jurídico vigente más relevante aplicable a la entidad, al directorio y sus ejecutivos principales.

El 100\% de las empresas, cuentan con un procedimiento de inducción que incluye material escrito y reuniones informativas.

- Los deberes de cuidado, reserva, lealtad, diligencia e información que conforme a la legislación vigente recaen en cada integrante del directorio, mediante ejemplos de fallos, sanciones o pronunciamientos más relevantes que han ocurrido en el último año a nivel local con esos deberes.

Las empresas en estudio dentro del procedimiento de inducción, en el cual el gerente de asunto legales de la compañía, da a conocer el marco jurídico vigente, pone énfasis en los deberes de cuidado, reserva, lealtad, diligencia e información que conforme a la legislación vigente recae en cada uno de los integrantes del directorio, al asumir el cargo.

- Las partidas más relevantes de los estados financieros trimestrales y anuales del último año junto con sus respectivas notas explicativas, además de los criterios contables aplicados en la confección de dichos estados financieros.

Las empresas cumplen con la entrega de dicha información, y en algunos de los casos es complementada con una reunión programada entre el nuevo integrante del directorio y la dirección de administración y finanzas de la compañía.

Las prácticas menos implementadas:

\section{b) El Directorio cuenta con un procedimiento o mecanismo de capacitación permanente del Directorio para la actualización de conocimientos, que:}

- $\quad$ Define al menos anualmente las materias respecto de las cuales se harán capacitaciones a sus integrantes y el calendario de capacitaciones para el año correspondiente.

Algunas de las empresas no cumplen con esta práctica, debido a que no la consideran necesaria, ya sea porque se asume que los integrantes del directorio debiesen estar ya capacitados al momento de asumir el cargo.

- Como parte de esas materias contempla los principales avances que se han dado en el último año a nivel local e internacional en lo referido a inclusión, diversidad y reportes de sostenibilidad.

- Como parte de esas materias contempla las principales herramientas de gestión de riesgos, incluidos los de sostenibilidad, que se han ido implementando en el último año a nivel local e internacional. 
- Como parte de esas materias contempla los fallos, sanciones 0 pronunciamientos más relevantes que han ocurrido en el último año a nivel local e internacional relacionados con los deberes de cuidado, reserva, lealtad, diligencia e información.

- Como parte de esas materias contempla una revisión de ejemplos de situaciones que configuran un conflicto de interés en el directorio y de formas en que esos conflictos de interés pueden evitarse o ser resueltos en el mejor interés social.

- $\quad$ Difunde anualmente las materias sobre las que en el último año se han realizado actividades de capacitación al directorio.

La mayoría de las empresas, no entregan explicación a su respuesta, otras señalan, que no consideran necesaria llevar a cabo esta práctica, o que la política para tratar este tema se encuentra en proceso de implementación.

\section{c) El Directorio cuenta con una política para la contratación de expertos(as) que lo asesoren en materias contables, tributarias, financieras, legales 0 de otro tipo:}

- Que contemple la posibilidad de veto por parte de uno 0 más directores para la contratación de un(a) asesor(a) en particular.

Si bien las empresas cuentan con políticas de contratación, esta señala que para la contratación de un asesor, se requiere de una sala legalmente constituida y con la participación de la mayoría establecida en la legislación vigente o por el quorum de la mayoría absoluta de los directores asistentes.

- Que a requerimiento de al menos uno de sus integrantes sea contratada la asesoría para la materia requerida por aquél.

Al igual que en el punto anterior, se señala que esta decisión le corresponde tomarla al directorio como órgano colegiado, con el quorum de la mayoría absoluta establecida en la legislación vigente.

- Que, contemple la difusión, al menos una vez al año, de las asesorías solicitadas y no contratadas, especificando las razones por las que el directorio adopto esa decisión en particular, lo cual además queda debidamente reflejado en el acta de la sesión correspondiente.

Esta práctica no aplica, debido a que la contratación de asesorías es una decisión del directorio en conjunto, no existe el motivo para que la contratación no se realice. Además de considera que la difusión de asesorías solicitadas y no contratadas no es un tema que agregue valor a la empresa.

d) El Directorio se reúne al menos trimestralmente con la unidad de gestión de riesgos de la entidad o responsable de función equivalente, para analizar: 
- $\quad$ El adecuado funcionamiento del proceso de gestión de riesgos.

- La matriz de riesgos empleada por la unidad así como las principales fuentes de riesgo y metodologías para la detección de nuevos riesgos y la probabilidad e impacto de ocurrencia de aquellos más relevantes.

- Las recomendaciones y mejoras que en opinión de la unidad sería pertinente realizar para gestionar de mejor manera los riesgos de la entidad.

- Los planes de contingencia diseñados para reaccionar frente a la materialización de eventos críticos, incluida la continuidad del directorio en situaciones de crisis.

Las empresas no cumplen estas 4 prácticas, principalmente por la periodicidad que se exige, pero la mayoría de las sociedades en estudio cuentan con un departamento de gestión de riesgo, dependiente de la gerencia corporativa de auditoria interna.

e) El Directorio se reúne al menos trimestralmente con la unidad de Responsabilidad Social, Desarrollo Sostenible o responsabilidad de función equivalente, para analizar:

- La efectividad de las políticas aprobadas por el directorio para difundir al interior de la organización, sus acciones y al público en general los beneficios de la diversidad e inclusión para la sociedad.

- Las barreras organizacionales, sociales o culturales detectadas que pudieren estar inhibiendo la natural diversidad que se habría dado de no existir esas barreras.

- La utilidad y aceptación que han tenido los reportes de sostenibilidad difundidos a los grupos de interés relevantes de la sociedad.

Estas prácticas no son cumplidas por las empresas en estudio, ya sea porque no existe una unidad de responsabilidad social en la entidad, o bien existiendo esta unidad, se reúne al menos trimestralmente con el gerente general corporativo y la alta administración para analizar estos temas.

\section{f) El Directorio cuenta con un procedimiento formal de mejoramiento continuo en operación:}

- Para detectar y reducir barreras organizacionales, sociales o culturales que pudieren estar inhibiendo la natural diversidad de capacidades, visiones, características y condiciones que se habría dado en el directorio de no existir esas barreras.

- Que, sin perjuicio de las obligaciones legales, contemple expresamente 
la determinación del número mínimo de reuniones ordinarias, el tiempo promedio mínimo de dedicación presencial y remota a las mismas, y la antelación con la que se debiera remitir la citación y los antecedentes necesarios para la adecuada realización de aquéllas, reconociendo las características particulares de la entidad así como también la diversidad de experiencias, condiciones y conocimientos existentes en el directorio, según la complejidad de las materias a tratar.

Debido al correcto funcionamiento del directorio que las empresas señalan tener, no consideran necesario contar con un procedimiento específico para tratar estas materias

\section{g) El Directorio cuenta con un sistema de información en operación y de acceso por parte de cada director que:}

- $\quad$ Permite la revisión a que se refiere el número v anterior, con no más de 5 días posteriores a la sesión respectiva.

Esta práctica no es cumplida por las sociedades en estudio, ya que, las empresas no cuentan con un sistema de información para acceder a información remota.

\section{De la relación entre la Sociedad, Ios Accionistas y el Público en General.}

Este grupo está formado por 22 prácticas, las cuales se refieren a las obligaciones de información tanto por parte de la sociedad, los accionistas mayoritarios, directores y ejecutivos, con el objeto de reducir la asimetría de información, el grafico muestra que el $68 \%$ de estas prácticas no son cumplidas por las empresas del sector retail analizadas.

Gráfico $N^{\circ}$ 4: Cumplimiento de prácticas de la relación entre la Sociedad, los Accionistas y el Público en General.

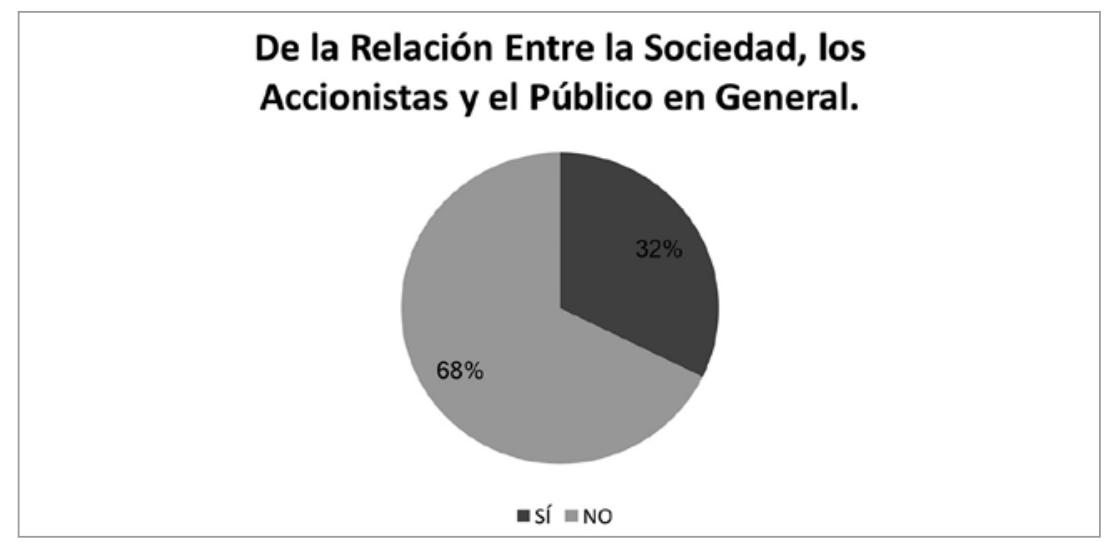

Fuente: Elaboración Propia. Año 2015.

Las prácticas en las cuales el $90 \%$ o más de las empresas coincidieron en las respuestas, son las siguientes: 
Gráfico $N^{\circ}$ 5: Prácticas relacionadas con la relación entre la Sociedad, los Accionistas y el Público en General.

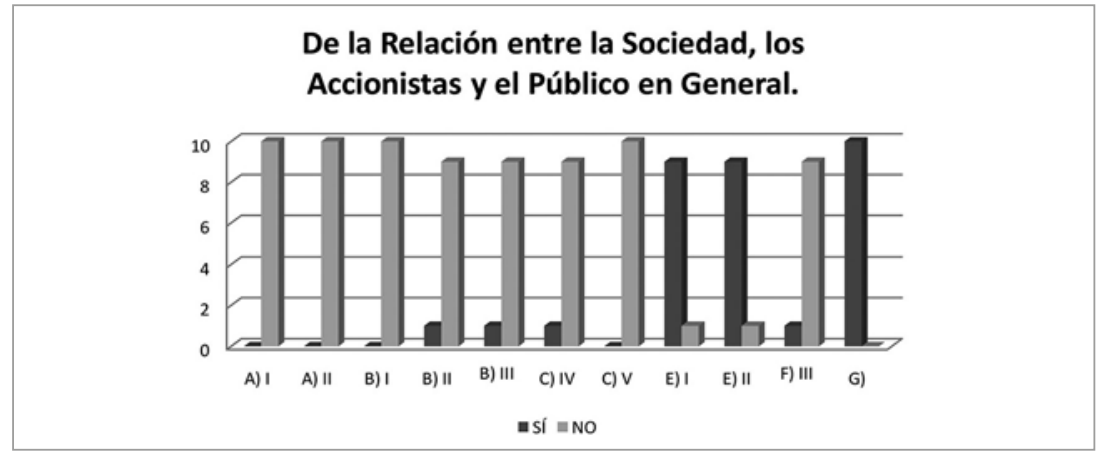

Fuente: Elaboración Propia. Año 2015.

Prácticas más implementadas:

e) La Sociedad cuenta con una unidad de relaciones con los accionistas, inversionistas y medios de prensa que:

- Permite a éstos aclarar dudas de la sociedad, sus negocios, principales riesgos, situación financiera, económica o legal y negocios públicamente conocidos de la entidad.

Las empresas cuentan con una gerencia de relaciones con inversiones encargada de responder de manera oportuna a las inquietudes planteadas por los accionistas e inversionistas de la sociedad, así como a los medios de prensa, respecto de la información pública de la sociedad, orientándolos para la obtención de la información que por ley puede ser provista a los accionistas y al público en general.

- Cuenta con personas que, al menos, dominen el idioma ingles para responder a las consultas de quienes no hablen español.

En las empresas en estudios aquellos que trabajan en la gerencia de relaciones con inversiones 0 el profesional que responda a las consultas, según sea el caso, dominan el idioma inglés.

g) La Sociedad cuenta con una página web actualizada por medio de la cual los accionistas pueden acceder a toda su información pública, de manera sencilla y de fácil acceso.

Las empresas cuentan con un sitio web, el que se encuentra permanentemente en proceso de revisión y actualización, y que permite un acceso fácil y rápido a accionistas y público en general, a fin de que conozca la información pública de la compañía.

Prácticas menos implementadas: 


\section{a) El Directorio ha implementado un procedimiento formal y en operación para que los accionistas de la sociedad se puedan informar:}

- Con al menos 3 meses de antelación a la junta de accionistas en que se elegirán directores, acerca de la diversidad de capacidades, condiciones, experiencias y visiones que en opinión del directorio resulta aconsejable formen parte del mismo para que éste esté en mejores condiciones de velar por el interés social.

Las empresas no consideran necesario contar con un procedimiento formal de definición de las características profesionales y personales de futuros integrantes del directorio en el sentido dispuesto con la norma.

- $\quad$ Antes de la votación correspondiente, del número máximo de directorios que, en opinión del directorio, es aconsejable tengan los directores que sean electos por los accionistas.

Las empresas no cuentan con políticas que señalen el número máximo de directorios que es aconsejable que los directores participen, pues se estima que cada uno de ellos lo debe definir de acuerdo al tiempo que, conforme a sus deberes de director, debe brindar a cada directorio que participe.

\section{b) El Directorio ha implementado un mecanismo, sistema o procedimiento formal que permita:}

- A los accionistas participar y ejercer su derecho a voto por medios remotos, en la misma oportunidad que el resto de los accionistas que están físicamente representados en la junta.

Las empresas no justifican la implementación de esta práctica, debido a los costos que significaría y principalmente por el alto quorum que registran las juntas de accionistas de las sociedades.

- A los accionistas observar, de manera remota y en tiempo real, lo que ocurre durante las juntas de accionistas.

Las sociedades no han implementado un mecanismo de este tipo, entregando la misma justificación que en el numeral anterior, por el alto número de participantes en las juntas de accionistas.

- $\quad$ Al público en general informarse en tiempo real de los acuerdos adoptados en la junta de accionistas.

Las empresas no estiman necesario implementar un mecanismo que permita al mercado y público general observar lo que ocurre en la junta de accionistas, sin embargo el directorio informa con la mayor celeridad los acuerdos adoptados en la junta de accionistas, relevantes para el mercado, a través de la página web de la sociedad y se envía a la Superintendencia de Valores y Seguro (SVS) los hechos 
esenciales.

c) El Directorio ha aprobado una política y establecido procedimientos formales que tienen por objetivo proveer anualmente al público información respecto a:

- $\quad$ Los indicadores medidos por la sociedad en materia de responsabilidad social y desarrollo sostenible.

- $\quad$ La existencia de metas y la evolución que han tenido los indicadores de sostenibilidad.

Las empresas no cumplen con estas dos prácticas, ya sea porque no cuentan con una unidad de responsabilidad social y desarrollo sostenible dentro de su estructura, el reporte sobre sostenibilidad social no incluye esta materia 0 no se considera necesario contar con una política de esta naturaleza.

f) El directorio cuenta con un procedimiento formal de mejoramiento continuo en operación:

- Que considere la asesoría de un experto ajeno a la sociedad para la detección e implementación de esas eventuales mejoras.

La mayoría de las sociedades no cuentan con este procedimiento y no considera necesario contar con el apoyo de un experto en esta materia, debido a que se ha considerado que tanto el Directorio como el Gerente General y su línea ejecutiva pueden cumplir dicha función, sin incurrir en los costos adicionales que supondrían tales asesorías.

\section{De la gestión y control de riesgo.}

El $45 \%$ de las 22 prácticas que conforman este grupo son cumplidas por las empresas del sector retail en estudio, lo cual quiere decir, que el $45 \%$ del total de las practicas relacionadas con los mecanismos, procedimientos, recursos, sistemas y controles que las sociedades han ido generando para hacer frente a los cambios de su entorno o las situaciones adversas que se les presenten, están siendo llevadas a cabo. 
Gráfico $\mathrm{N}^{0}$ 6: Cumplimiento de prácticas relacionadas con la gestión y control de riesgo.

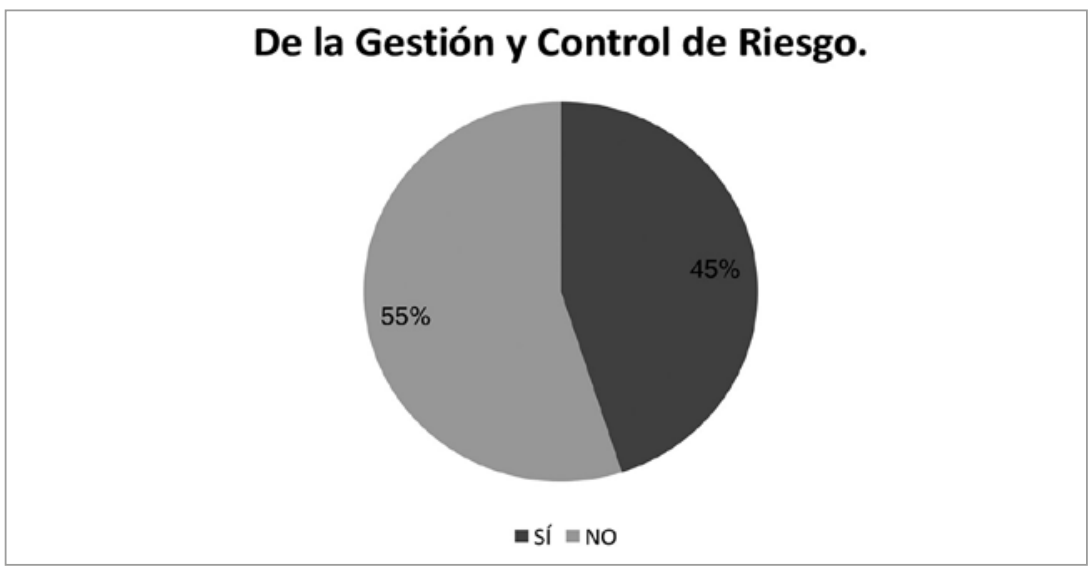

Fuente: Elaboración Propia. Año 2015.

Las prácticas en las cuales el $90 \%$ o más de las empresas analizadas coincidieron en su respuesta, se muestran en el siguiente gráfico.

Gráfico $\mathrm{N}^{0} 7$ : Prácticas relacionadas con la gestión y control de riesgo.

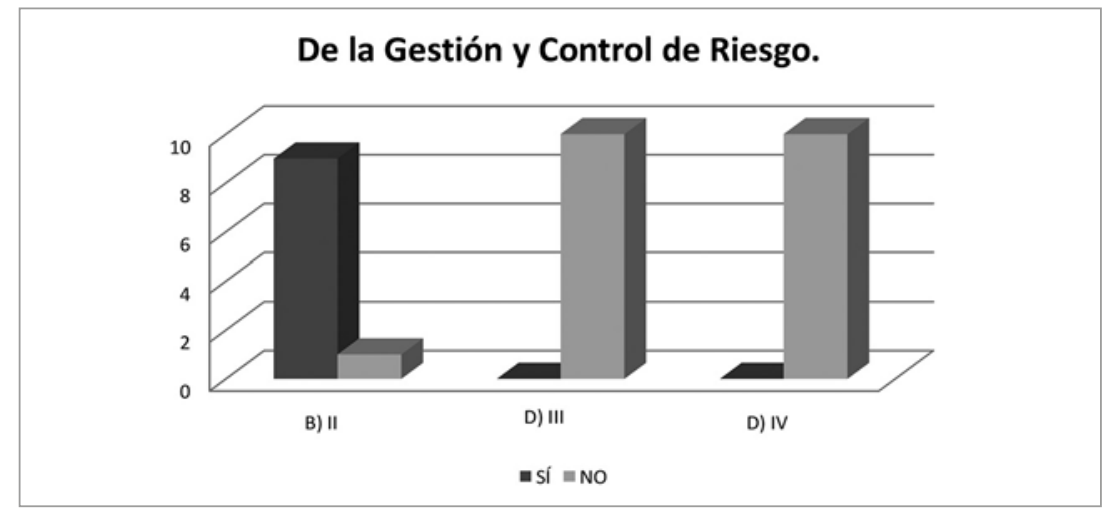

Fuente: Elaboración Propia. Año 2015.

Práctica más implementada:

b) El Directorio ha implementado un procedimiento formal y que está en operación:

- Que garantiza el anonimato del denunciante.

Las empresas cuentan con una línea de denuncia, la cual es administrada por un tercero independiente, que permite que la denuncia sea anónima y asegura la confidencialidad de los denunciantes, si este así lo solicita. 
Prácticas menos implementadas:

d) El Directorio ha implementado un procedimiento formal y que está en operación:

- Que contempla la difusión de las estructuras salariales y políticas de compensación e indemnización del gerente general y demás ejecutivos principales, en el sitio en Internet de la sociedad.

Las sociedades no han contemplado la difusión de estructuras salariales y políticas de compensación e indemnización del gerente general y demás ejecutivos principales.

- Que contempla someter dichas estructuras salariales y políticas a aprobación de los accionistas.

Las empresas no han contemplado someter las estructuras salariales del Gerente y ejecutivos principales a la aprobación de los accionistas, debido a que esta es una facultad del directorio de la sociedad.

\section{De la evaluación por parte de un tercero.}

Las prácticas relacionadas con la evaluación por parte de un tercero son cuatro, donde el $98 \%$ de las empresas respondió que no efectúa ninguna de estas prácticas, es decir, no se está entregando información confiable respecto al cumplimiento de las prácticas de fortalecimiento de gobierno corporativo.

\section{Gráfico № 8: Cumplimiento de prácticas relacionadas con la evaluación por parte de un tercero.}

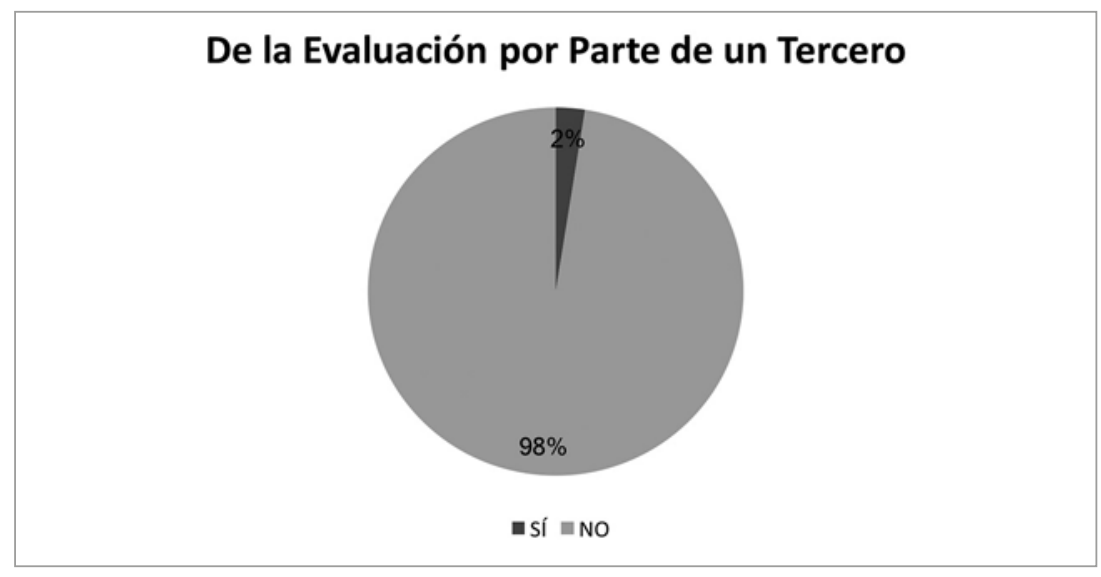

Fuente: Elaboración Propia. Año 2015.

Estas cuatro prácticas son analizadas a continuación. 
Gráfico № 9: Prácticas relacionadas con la evaluación por parte de un tercero.

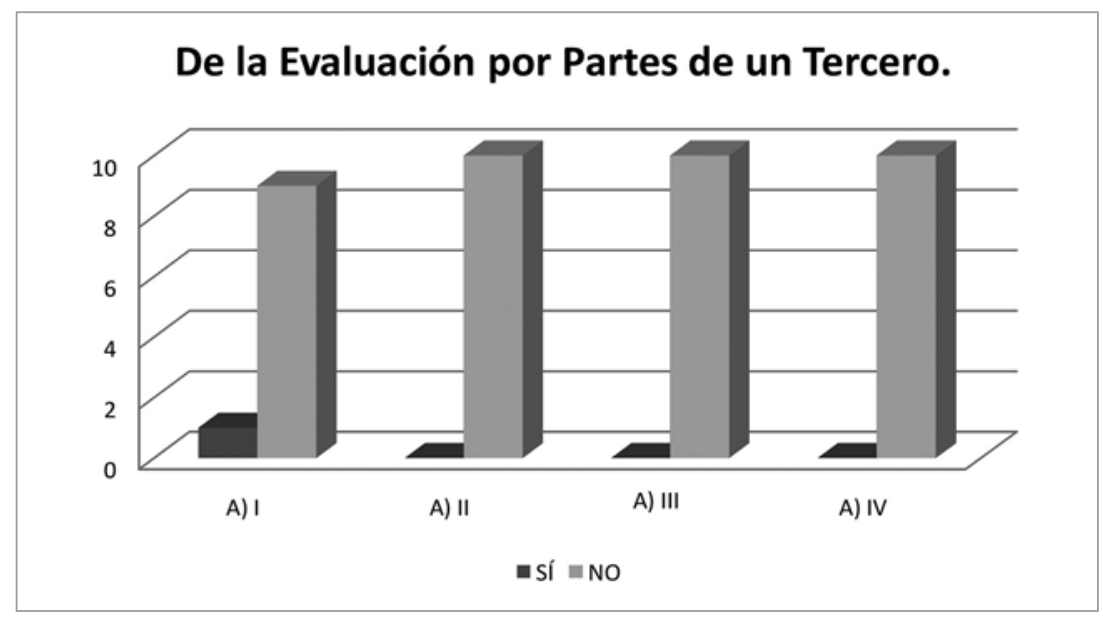

Fuente: Elaboración Propia. Año 2015.

a) La autoevaluación del directorio respecto a la adopción de las prácticas contenidas en la presente normativa:

- Ha sido revisada y validada por un tercero ajeno a la sociedad.

- La persona 0 equipo de personas que realizaron la revisión y validación, cuenta con experiencia acreditada de al menos 5 años en evaluación de procesos y efectividad de controles, 0 en la prestación de servicios profesionales de asesoría o consultoría en diseño e implementación de procesos, gestión de riesgos o mejoramiento continuo.

- La entidad o personas que realizaron la revisión y validación, son fiscalizados por la Superintendencia u organismo público 0 privado extranjero de similar competencia.

- La entidad o personas que realizaron la revisión y validación, pertenecen a una nómina establecida por las bolsas nacionales de entidades que cumplen las condiciones definidas por las mismas para certificar la autoevaluación a que se refiere la presente normativa.

Las empresas en estudio entregan la misma respuesta para las 4 prácticas, con excepción de Ripley quien señala que la autoevaluación del directorio respecto a la adopción de las prácticas contenidas en la presente normativa.

El resto de las empresas no consideran necesario la revisión por parte de un tercero, les es suficiente con una revisión interna. 


\section{DISCUSIÓN DE RESULTADOS}

El primer punto a discutir trata el efecto del reemplazo de la norma de carácter general (NCG) № 341 por la Norma de Carácter General (NCG) № 385. La Superintendencia de Valores y Seguros (SVS), señala que esta derogación efectuada el 8 de junio del 2015, se realiza con la finalidad de reordenar y desagregar las prácticas de Ggobierno Corporativo. En la investigación se muestra que tal finalidad no es completamente alcanzada, si bien las empresas respetan el formato entregado en el propio anexo de la norma, pero, las empresas entregan las mismas respuestas a aquellas prácticas relacionadas como si fuera una sola.

En segundo lugar, se discute sobre la aplicación de la Norma de Carácter General (NCG) No 385 en las sociedades anónimas del sector retail, si bien, la misma norma señala que tanto su aplicación, como el cumplimiento de las prácticas, no tiene el carácter de obligatorio, la investigación muestra que el 76,92\% de las empresas del sector en estudio, aplicaron esta norma, siendo la excepción $A D$ Retail S.A., Plaza S.A. y Sodimac S.A.

La organización para la cooperación y desarrollo económico (OCDE), sostiene que el gobierno corporativo es el mecanismo de los accionistas que sirve de contrapeso y guía a la administración del negocio, para asegurar la eficiencia, la equidad, la transparencia y los buenos resultados, con razonabilidad, rendición de cuentas, transparencia y responsabilidad.

La discusión recae en que si este gobierno corporativo funciona de acuerdo a la definición entregada. La norma de Carácter General busca establecer las prácticas que fortalezcan estas funciones separándolas en 4 grupos.

1. Del funcionamiento y composición del directorio. La mayoría de las empresas en estudio cuentan con un procedimiento de inducción, el que consiste en reuniones con los principales gerentes de la compañía y la entrega de la información más relevante de la entidad, por lo cual se podría decir que las empresas de retail en Chile, que reportan a la SVS, están en condiciones de hacer frente a las contingencias y riesgos que pueden presentarse.

2. De la relación entre la sociedad, los accionistas y el público en general. Las empresas en estudio cuentan con una gerencia de inversiones encargadas de responder de manera oportuna las inquietudes planteadas respecto de la información pública de la sociedad a los accionistas e inversionistas de la sociedad y al medio de prensa. La información también se encuentra disponible en las páginas web de las empresas, la cual es actualizada permanentemente. Pero no tienen implementado un mecanismo que permita conocer en tiempo real los acuerdos adoptados en la junta de accionista y tampoco permite a los accionistas ejercer su derecho a voto de forma remota, debido a los costos que significarían a 
la empresa implementarlo, y no siendo justificado, debido al alto quorum participante en la junta de accionista.

3. De la gestión y control de riesgo. En las empresas en estudio, las prácticas que más implementan para el fortalecimiento de sus gobiernos corporativos, son las relacionadas con la gestión y control de riesgo, dentro de las cuales sobresale el contar con un canal de denuncias, el cual resguarda el anonimato del denunciante cuando así este lo requiere. En el otro extremo, las entidades no difunden la información salarial de los gerentes y ejecutivos principales, por considerarlo como información personal de la empresa.

4. De la evaluación por parte de un tercero. Las entidades analizadas, poseen un riesgo de cumplimiento alto, en esta materia, debido a que todas las empresas señalan que no cumplen con las prácticas relacionadas con la evaluación de un tercero, es decir, la autoevaluación realizada por el directorio respecto a la adopción de las practicas contenidas en la Norma de Carácter General № 385 , no es revisada y revaluada por un tercero ajeno a la sociedad, por ser considerado por las empresas, que sus directorios tienen las competencias suficientes para realizar por si solos esta autoevaluación.

\section{CONCLUSIONES}

Basado en la información obtenida en esta investigación, se puede concluir que la mayoría de las empresas de Retail, reguladas por la SVS, cumplen con emitir el Informe sobre Prácticas de Gobierno Corporativo a pesar de no ser algo obligatorio, pero algunas de las empresas responden al "cuestionario" establecido en la Norma de Carácter General (NCG) № 385, como un mero trámite, debido a que principalmente Ripley, en la mayoría de las prácticas analizadas, no entrega observaciones a sus respuestas, siendo que lo más importante para los usuarios de estos informes no son las preguntas, si no que las respuestas, las justificaciones del porque cumplen o no cumplen con las prácticas señaladas.

Por otra parte, las empresas respondieron a varias prácticas de manera negativa, por no ser una obligación legal, lo que lleva a suponer que su funcionamiento no lo basan en obtener mejores beneficios para las mismas empresa, como en este caso se persigue el fortalecimiento de los gobiernos corporativos, si no, que solo cumplir por el hecho de que existe una ley que lo exige y la cual sanciona en caso de su no cumplimiento, es decir, las empresas del sector retail funcionan "correctamente" por mera obligación.

También se puede señalar que a pesar de que hoy en día las Sociedades Anónimas Abiertas, entregan una gran cantidad de información públicamente, para que esta pueda ser utilizada por los accionistas, inversionistas y el público en general, las 
empresas aún están negativas a dar a conocer cierta información, consideradas por ellas como confidencial de la empresa, la cual la Norma de Carácter General considera que al ser publicada puede fortalecer a sus gobiernos corporativos.

Las prácticas que más cumplen las empresas de retail, son las relacionadas con el control y gestión de riesgo, lo cual además de proteger a la sociedad, de los cambios de su entorno 0 de situaciones adversas que se les presenten; también da garantías a los inversionistas, reguladores y demás grupos de interés, que habrá una menor probabilidad de que la entidad se vea envuelta en fraudes o en situaciones que puedan afectar la confianza de los mercados, finalidad que se persigue con la implementación de gobierno corporativo en las empresas.

En la parte contraria, aquellas prácticas que presenta mayor cantidad de respuestas negativas (98\%), corresponde al último grupo de prácticas para el fortalecimiento de estándares de gobierno corporativo, correspondiente a la evaluación por parte de un tercero, no estaba incluido dentro de la Norma de Carácter General No 341 , solo fue evaluado a partir del año 2015, con la emisión de la nueva Norma de Carácter general № 385 , lo que lleva a concluir que las empresas no están implementando nuevas prácticas que le permita un mejor funcionamiento de sus gobiernos corporativos, no están analizando a fondo que lo que se pretende lograr con esta práctica es una mayor confianza por parte de usuarios interesados en esta información, de que lo que el directorio está mostrando es la realidad de la empresa, debido a que la información es revisada y respaldada por una persona independiente de la empresa, y no es algo innecesario como estas señalan en sus respuestas.

\section{BIBLIOGRAFÍA}

- DFL 1 https://www.leychile.cl/Navegar?idNorma=172986 (Visitado el 13/10/2016)

- Desarrollo de una metodología para el análisis del comportamiento de la industria del retail en Chile. http://repositorio.uchile.cl/bitstream/ handle/2250/103778/cf-lucchini_fa.pdf?sequence $=3 \&$ isAllowed $=y$ (Visitado el 29/10/2016)

- Evolución del Gobierno Corporativo de las sociedades anónimas locales y el rol de la Bolsa http://www.bolsadesantiago.com/Biblioteca\%20BCS/ Charla\%20Gobiernos\%20Corporativos,\%20Juan\%20Andr\%C3\%A9s\%20 Camus.pdf (visitado el 09/05/2016)

- Fortaleciendo el estándar de los gobiernos corporativos en Chile https:// www.svs.cl/portal/prensa/604/articles-21653_doc_pdf.pdf (Visitado el 08/11/2016) 
- Gobierno Corporativo en Chile después de la ley de Opas http://www.dii. uchile.cl/ ceges/publicaciones/ceges38.pdf (Visitado el 05/05/2016)

- Gobiernos Corporativos en Chile: Una mirada a la implementación de la Norma de Carácter General №385

- https://www.pwc.com/cl/es/publicaciones/assets/2016/Estudio-NCG385-2-VF.pdf (Visitado el 27/07/2016)

- Ley 18046 http://www.leychile.cl/Navegar?idNorma=29473 (Visitado el 13/10/2016)

- Leyton Luis, 2013. Revista Retailing (Visitado el 29/10/2016)

- Organización para la Cooperación y Desarrollo Económico (2015) http://www.elmostrador.cl/noticias/opinion/2015/11/23/los-gobiernoscorporativos/ (Visitado el 29/04/2016)

- Norma de Carácter General №341; https://www.svs.cl/normativa/ ncg_341_2012.pdf (Visitado el 27/07/2016)

- Norma de Carácter General № 385; http://www.svs.cl/normativa/ ncg_385_2015.pdf (visitado el 27/07/2016)

- Wigodski Teodoro y Zuñiga Franco. 2001. Gobierno Corporativo en Chile. Tesis para optar al grado de magister de Gestión y Dirección de Empresas. Departamento de Ingeniería Industrial. Universidad de Chile. Santiago). 